\title{
Observation des spermatozoïdes au fort grossissement (MSOME) : intérêt et perspectives
}

\author{
Martine ALBERT1, 2, Mohamed Hassen CHELLI ${ }^{1}$, Nathalie SERMONDADE1, \\ Marianne BERGERE1, 2, Ibrahim HAMMOUD1, 2, Jacqueline SELVA1, 2
}

1 Service d'Histologie-Embryologie-Cytogénétique, Biologie de la Reproduction et Génétique Médicale. C.H.I. Poissy-Saint Germain en Laye

2 UFR Paris Île de France Ouest, Université Versailles - Saint Quentin en Yvelines

\section{INTRODUCTION}

La morphologie des spermatozoïdes observée en microscopie optique conventionnelle est indéniablement un paramètre fondamental de la qualité du sperme. Les anomalies qui sont ainsi détectées peuvent avoir des répercussions sur la fonction des spermatozoïdes et un impact sur leur aptitude migratoire et fécondante en reproduction naturelle, en IIU ou en FIV. Certaines anomalies sont repérées à l'évidence à la lecture du spermocytogramme (macrocéphalie, microcéphalie, globozoospermie), mais d'autres anomalies sont plus subtiles... Quoiqu'il en soit, lorsque la morphologie est très altérée, l'ICSI est en général la solution thérapeutique adoptée, souvent avec succès, même dans des cas de tératospermie totale.

Cependant, que l'indication de l'ICSI soit une tératospermie importante, associée ou non à une altération des autres paramètres ou à un échec de fécondation préalable inexpliqué en FIV, les résultats de I'ICSI sont parfois décevants : le taux de fécondation est faible ou nul, la qualité embryonnaire défectueuse, le taux d'implantation abaissé ou nul, voire le taux de Beta hCG chute très rapidement.

Dans ce contexte d'échec d'ICSI, l'approche morphologique des spermatozoïdes à un fort grossissement proposée par Bartoov et al. [1] et appelée MSOME (Motile Sperm Organelle Morphology Examination) a dès lors constitué un progrès considérable en permettant de détecter sur cellules vivantes, avec un grossissement allant jusqu'à plus de $X 10.000$, des anomalies non visualisables par les autres techniques. Cette technique a été d'emblée appliquée à la sélection du spermatozoïde injecté en ICSI qui prend alors le nom d'IMSI (Intracytoplasmic Morphologically Selected Sperm Injection) [3]. Ces dernières années, cette méthode s'est développée dans d'autres centres d'Assistance Médicale à la Procréation, qui, pour la plupart, témoignent parfois d'un bénéfice quant au taux de fécondation [4], mais surtout d'un bénéfice constant concernant les taux d'implantation, de grossesse, d'accouchement ainsi qu'une diminution des taux de fausses couches spontanées $[5,6,9]$.

\section{QU'EST CE QUE LE MSOME EN PRATIQUE?}

L'observation à fort grossissement de spermatozoïdes vivants nécessite un système optique particulier, à savoir, un microscope inversé, doté d'un objectif x100 pour le contraste interférentiel de Nomarsky ( \pm d'un zoom variable), une caméra vidéo numérique couleur de bonne qualité ( \pm d'un zoom variable), et un moniteur couleur de haute définition. L'ensemble de ces systèmes optiques permet une observation des spermatozoïdes à un grossissement allant de l'ordre de 2000 à 12500 fois selon les configurations spécifiques. Toutefois, les contraintes techniques sont importantes, principalement liées au matériel utilisé, à la préparation

Correspondance :

Dr Martine ALBERT - Service d'Histologie-EmbryologieCytogénétique, Biologie de la Reproduction et Génétique Médicale, C.H.I. Poissy-Saint Germain en Laye, 78303 Poissy cedex - Email martine_albert@hotmail.com 
de la boite de sélection, à la qualité du sperme observé et à l'éventuelle injection ultérieure du spermatozoïde sélectionné. L'expérience de la technique et le fort grossissement utilisé montrent qu'une observation en MSOME est facilement réalisable quand on dénombre entre 1 et 20 spermatozoïdes par champ au grossissement $\times 6000$, idéalement de l'ordre d'une dizaine.

L'observation en MSOME est difficile dans les cas de cryptozoospermie et d'oligozoospermie extrême. La durée totale de la technique varie de 30 minutes à $2 \mathrm{~h} 30$, selon la qualité de l'échantillon et l'expérience de l'opérateur au grossissement $\times 6000$. Si la finalité du MSOME est l'IMSI, la sélection morphologique est le temps le plus important et souvent le plus long de la technique, notamment en cas de tératozoospermie sévère.

\section{QUELLES SONT LES ANOMALIES OBSER- VÉES EN MSOME ?}

Dans notre centre, nous avons dans un premier temps tenté de recenser dans des préparations de spermatozoïdes migrés les différentes anomalies observables en MSOME, dans le but de disposer d'un atlas à visée didactique, référentiel pour l'ensemble des utilisateurs du laboratoire, et d'élaborer une grille de lecture rigoureuse.

En dehors des anomalies de la tête spermatique aisément reconnaissables en MSOME telles que les anomalies de taille, de forme, de régularité du pourtour des têtes, nous avons noté deux particularités : (1) la moindre lisibilité des acrosomes et à l'inverse, (2) l'excellente lisibilité des vacuoles localisées dans la région céphalique. En ce qui concerne les flagelles, l'observation au fort grossissement est d'autant plus difficile que les spermatozoïdes sont mobiles, difficulté contournable par l'utilisation d'une solution visqueuse. Les différentes anomalies de la pièce intermédiaire et de la pièce principale sont bien visualisées.

Au terme de cette étude préliminaire détaillée des anomalies observables en MSOME, nous avons ajouté à la grille élaborée par Bartoov et al. [2] trois critères : contour irrégulier et anomalie régionale au niveau de la tête, irrégularité de la pièce intermédiaire. La rubrique " vacuoles " a également été détaillée par le nombre de vacuoles observées, l'estimation de leur surface (rapportée à celle de la tête spermatique) et leur localisation (région antérieure ou postérieure de la tête) [10]. La signification de ces vacuoles et leur support ultrastructural à l'échelle de la cellule seront discutés.

\section{QU'EST CE QUE LE MSOME APPORTE AU PLAN DIAGNOSTIQUE MORPHOLOGIQUE ?}

A l'évidence, le grossissement obtenu en MSOME permet de visualiser des anomalies des spermatozoïdes migrés qui ne l'auraient pas été avec les grossissements habituels. Ces différences concernent surtout les anomalies non répertoriées au spermocytogramme conventionnel, anomalie régionale et vacuoles:[10]. De plus, un pourcentage notable des spermatozoïdes pré-sélectionnés pour une ICSI conventionnelle sont en fait anormaux en MSOME.

\section{QUEL EST L'INTÉRÊT DU MSOME AU PLAN THÉRAPEUTIQUE ?}

La technique d'observation des spermatozoïdes au fort grossissement a été d'emblée appliquée à l'ICSI [1] avec des taux de grossesse clinique avoisinant les $60 \%$, et même supérieurs lorsque l'IMSI était réalisée avec des spermatozoïdes strictement normaux. Ces résultats se sont confirmés au fil des études, la normalité du noyau dans sa forme et son contenu étant corrélée au taux de fécondation en IMSI [2, 3]. Berkovitz et al. [4] confirment ces résultats, précisant que le taux de fécondation, le nombre de " top embryons ", le taux d'implantation, le taux d'accouchement allaient de pair avec l'injection de spermatozoïdes strictement normaux en MSOME, réduisant ainsi le taux de FCS à un taux très bas (de l'ordre de $5 \%$ ).

Ces résultats soulèvent le problème de mieux définir les anomalies morphologiques du noyau impliquées dans les échecs. Berkovitz et al. [5] ont ainsi montré que les vacuoles de grande taille $(>0,78 \mu \mathrm{m})$ étaient sans effet sur le taux de fécondation mais affectaient le taux de grossesse clinique (donc augmentaient la mort embryonnaire précoce).

Une autre approche a consisté à corréler les résultats du MSOME avec d'autres investigations spermatiques, comme par exemple le taux de fragmentation de l'ADN spermatique $[7,9]$ ou le taux d'aneuploïdie spermatique chez des patients à risque élevé tels que des hommes porteurs de translocations constitutionnelles, d'un caryotype $47, X Y Y$ ou présentant une macrocéphalie spermatique syndromique [8].

\section{DISCUSSION ET PERSPECTIVES}

L'intérêt majeur du MSOME réside donc dans la possibilité d'observer à fort grossissement les spermatozoïdes, de sélectionner ceux qui sont exempts de toute anomalie pour les microinjecter dans des ovocytes, et les auteurs témoignent tous de taux de succès en IMSI qui sont très élevés, notamment concernant les taux d'implantation, de grossesse clinique et d'accouchement. Ces résultats en IMSI sont d'autant meilleurs que l'on a pu sélectionner et injecter un spermatozoïde parfait au fort grossissement.

Ces résultats incontestables ouvrent des perspectives très intéressantes sur les relations à établir entre le phénotype du spermatozoïde injecté et son pouvoir fécondant et de développement embryonnaire. Car, au-delà 
de ces succès en IMSI, quelles sont, compte tenu des contraintes techniques évoquées, les indications les plus pertinentes pour proposer un MSOME et/ou une IMSI ? Qu'est-ce qui fait qu'un " top " spermatozoïde est meilleur qu'un autre ? Qu'y a-t-il de déterminant dans cette équation structure-fonction ? Qu'en est-il des spermatozoïdes imparfaits? De quels défauts cachés s'agit-il ? Concernent-ils l'intégrité nucléaire, la condensation de la chromatine, des cassures de l'ADN ? Avec ces interrogations, c'est aussi toute la question de l'effet paternel du spermatozoïde sur la fécondation et les premiers stades de développement embryonnaire qui est posée.

Au plan diagnostique, face à l'hétérogénéité de la population spermatique, le MSOME ouvre la perspective de pouvoir étudier avec nos outils habituels, des populations morphologiquement homogènes de spermatozoïdes normaux ou présentant une anomalie ciblée. Cela impose le tri des spermatozoïdes et l'adaptation de nos procédures techniques (quelles qu'elles soient) à des quantités restreintes de spermatozoïdes. De telles investigations et l'analyse morphologique fine de chaque spermatozoïde injecté associée au suivi de chaque ovocyte, puis le suivi embryon par embryon, permettraient en partie de répondre à ces questions et de progresser dans la relation structure fonction du spermatozoïde et le choix du spermatozoïde à injecter.

\section{REFERENCES}

1. BARTOOV B., BERKOVITZ A., ELTES F. et al. : Selection of spermatozoa with normal nuclei to improve the pregnancy rate with intracytoplasmic sperm injection. N. Engl. J. Med., 2001, 345 : 1067-1068.

2. BARTOOV B., BERKOVITZ A., ELTES F. et al. : Real-time fine morphology of motile human sperm cells is associated with IVF-ICSI outcome. J. Androl., 2002, 23 : 1-8.

3. BARTOOV B., BERKOVITZ A., ELTES F. et al. : Pregnancy rates are higher with intracytoplasmic morphologically selected sperm injection than with conventional intracytoplasmic injection. Fertil. Steril., 2003, $80:$ 1413-1419.

4. BERKOVITZ A., ELTES F., YAARI S. et al. : The morphological normalcy of the sperm nucleus and pregnancy rate of intracytoplasmic injection with morphologically selected sperm. Hum. Reprod., 2005, 20 : 185-190.

5. BERKOVITZ A., ELTES F., ELLENBOGEN A. et al. : Does the presence of nuclear vacuoles in human sperm selected for ICSI affect pregnancy outcome ? Hum. Reprod., 2006, $21: 1787-1790$.

6. BERKOVITZ A., ELTES F., LEDERMAN H. et al. : How to improve IVF-ICSI outcome by sperm selection ? Reprod. Biomed. Online, 2006, $12: 634-638$.

7. BOUGHALI H., WITTEMER C., VIVILLE S. et al. : Intérêt de l'analyse de la morphologie fine et de la qualité nucléaire des spermatozoïdes dans la cadre des techniques d'AMP. Andrologie, 2006, $16: 38-45$.
8. CHELLI M.H. : La sélection des spermatozoïdes à fort grossissement permet-elle une diminution de la fréquence des aneuploïdies spermatiques ? Mémoire de Master Pro BMDR, Université Paris V, 2007.

9. HAZOUT A., DUMONT-HASSAN M., JUNCA A.M. et al. : High-magnification ICSI overcomes paternal effect resistant to conventional ICSI. Reprod. Biomed. Online, 2006, 12 : $19-25$.

10. SERMONDADE N., VIALARD F., BERGERE M. et al. : Evaluation de l'apport de la méthode d'observation des spermatozoïdes à fort grossissement en ICSI. Andrologie, 2007, $17: 212-222$.

Manuscrit reçu : septembre 2007 ; accepté septembre 2007. Communication au XXVlème Congrès de la SALF, décembre 2007, Colmar 